\title{
Microbial and Antibiotic Susceptibility Profile among Isolates of Clinical Samples of Cancer Patients Admitted in the Intensive Care Unit at Regional Tertiary Care Cancer Center: A Retrospective Observational Study
}

\author{
Vishnu Kumar Garg ${ }^{1}$, Mishra Seema ${ }^{2}$, Gupta Nishkarsh ${ }^{3}$, Rakesh Garg ${ }^{4}$, Bharti Sachidanand ${ }^{5}$, Kumar Vinod ${ }^{6}$, \\ Hitender Gautam ${ }^{7}$, Arti Kapil ${ }^{8}$, Sushma Bhatnagar ${ }^{9}$
}

\begin{abstract}
Cancer patients in intensive care unit (ICU) are vulnerable for developing multidrug resistant nosocomial infections. The antimicrobial resistance due to inappropriate use of antibiotics results in significant morbidity and mortality in these cancer patients. The present retrospective study was done to describe the antimicrobial sensitivity pattern of common organisms in isolates of clinical samples of patients admitted in ICU at our tertiary care cancer center.

Materials and methods: The study was carried out at ICU of a regional tertiary care cancer center for a period of 1 year from October 2016 to September 2017. All clinical samples were collected and processed for culture and antibiotic susceptibility testing were carried out on isolates as per Clinical Laboratory Standard Institute guidelines.

Results: A total of 644 specimens were collected. Escherichia coli, Acinetobacter spp., Klebsiella pneumoniae, Pseudomonas aeruginosa, Staphylococcus aureus and Enterococcus spp. were most commonly encountered. In positive bacterial cultures, majority were Gram-negative isolates (84.14\%). Klebsiella was the most common gram-negative isolate (34.78\%) and Enterococcus spp. were the most common Gram-positive isolates (61.53\%). A high level of resistance to various antibiotics was noted among Gram-negative bacteria compared to Gram-positive isolates. Majority of the Gram-negative isolates were sensitive to Imipenem, Meropenem, and Colistin sensitivity among Gram-negative isolates was $100 \%$. Linezolid, Teicoplanin and Vancomycin were most sensitive antimicrobials against the Gram-positive bacteria.

Conclusion: Regular monitoring of the pattern of resistance of bacteriological isolates in cancer patients is critical to develop antibiotic policy to combat these infections and reduce morbidity and mortality.

Keywords: Antibiotics, Antibiotic susceptibility, Cancer, Culture, Multidrug resistance

Indian Journal of Critical Care Medicine (2019): 10.5005/jp-journals-10071-23119
\end{abstract}

\section{INTRODUCTION}

$M$ ultidrug resistant nosocomial infections are one of the leading causes of morbidity and mortality among hospitalized patients diagnosed with cancer ${ }^{1,2}$. There are usually many risk factors for acquiring infection in these cancer patients, such as long-term catheterization, mucositis due to cytotoxic agents, neutropenia and stem cell transplantation.

Patients in intensive care unit (ICU) are most vulnerable for developing these infections ${ }^{3}$. An ICU patient has five- to sevenfold higher incidence rate of nosocomial infection compared to the general inpatient population and ICU infections contributes to $20-25 \%$ of all nosocomial infections in a hospital ${ }^{4}$. This is due to the increasing use of invasive devices, such as mechanical ventilators, monitoring devices, blood and urine catheters, immunosuppressive drugs as well as irrational use of broad-spectrum antibiotic therapy in $I C U s^{3,5}$.

Empirical treatment of infections in the cancer patient is often attempted by administration of broad spectrum or combination antibiotics till the culture and susceptibility results are available.

The inappropriate and irrational use of antibiotics for therapeutic and nontherapeutic use leads to increasing antimicrobial resistance (AMR). In hospital care, this translates into prolonged hospital stay,

\begin{abstract}
${ }^{1-3}$ Department of Onco-Anaesthesia and Palliative Medicine, Dr BR Ambedkar, IRCH, All India Institute of Medical Sciences, New Delhi, India

${ }^{4-9}$ Department of Microbiology, All India Institute of Medical Sciences, New Delhi, India
\end{abstract}

Corresponding Author: Seema Mishra, Department of Oncoanesthesia and Palliative Medicine, IRCH, All India Institute of Medical Sciences, New Delhi, India, Phone: 9899061105, e-mail: seemamishra2003@gmail.com

How to cite this article: Garg VK, Seema M et al. Microbial and Antibiotic Susceptibility Profile among Isolates of Clinical Samples of Cancer Patients admitted in the Intensive-care Unit at Regional Tertiary Care Cancer Center: A Retrospective Observational Study. Indian J of Crit Care Med 2019;23(2):67-72.

Source of support: Nil

Conflict of interest: None

significant increase in morbidity and mortality and increasing economic burden on the individual and the nation.

The strains of multidrug resistant organisms have become four times worldwide, in recent years ${ }^{6}$. The rising trends of antibiotic resistance in commonly implicated organisms all over the world further enhance the risk of bacterial infections.

(c) The Author(s). 2019 Open Access This article is distributed under the terms of the Creative Commons Attribution 4.0 International License (http://creativecommons. org/licenses/by/4.0/), which permits unrestricted use, distribution, and non-commercial reproduction in any medium, provided you give appropriate credit to the original author(s) and the source, provide a link to the Creative Commons license, and indicate if changes were made. The Creative Commons Public Domain Dedication waiver (http://creativecommons.org/publicdomain/zero/1.0/) applies to the data made available in this article, unless otherwise stated. 
The profile of bacteria causing infections and their antibiotic sensitivity pattern vary widely from one geographical region to another as well as from one hospital to another and even among the ICUs within one hospital. Therefore, if the clinician has adequate information of the spectrum of microorganisms and the AMR patterns prevalent in that particular setting, appropriate empiric antibiotic therapy can be started.

The present retrospective study was done to describe the antimicrobial sensitivity pattern of common organisms in isolates of clinical samples of patients admitted in ICU of a tertiary care cancer center in India.

\section{Materials and Methods}

We conducted a retrospective, observational study in the ICU of a tertiary care cancer center in India for a period of 1 year from October 2016 to September 2017. The study was conducted after due approval obtained from the Institutional Ethical Committee (IEC-335/01.06.2018).

The study population included patients with various malignancies from medical, surgical and radiation oncology units undergoing treatment in our intensive care unit who had cultures sent for various reasons during study period.

Sample processing, identification of organisms to the species level and antimicrobial susceptibility tests were carried out as per Clinical and Laboratory Standards Institute (CLSI) guidelines, $2016^{7}$. Antimicrobial sensitivity patterns of respective organisms were studied on Mueller Hinton Agar (MHA) media by KirbyBauer's disk diffusion method ${ }^{8}$. Commercially available discs (HiMedia Laboratories, Mumbai, Maharashtra, India) were used and placed on the surface of the inoculated media and then incubated overnight. Zones of inhibition were measured the next day and were correlated with CLSI interpretive breakpoints to characterize them as sensitive, intermediate, and resistant. For drugs, such as colistin, for which CLSI breakpoints are not available, sensitivity were determined by MIC (minimum inhibitory concentration) method, with E-test strips in accordance with the European Committee on Antimicrobial Susceptibility Testing (EUCAST) guidelines ${ }^{9}$.

Antibiotics to be tested and reported for sensitivity against Gram-negative bacteria were ampicillin $(10 \mu \mathrm{g})$, gentamicin $(10 \mu \mathrm{g})$, amikacin $(30 \mu \mathrm{g})$, amoxycillin-clavulanic acid $(20 / 10 \mu \mathrm{g})$, cefotaxime $(30 \mu \mathrm{g})$, ceftriaxone $(30 \mu \mathrm{g})$, ceftazidime $(30 \mu \mathrm{g})$, cefoperazonesulbactam $(75 / 25 \mu \mathrm{g})$, ciprofloxacin $(5 \mu \mathrm{g})$, levofloxacin $(5 \mu \mathrm{g})$, norfloxacin $(5 \mu \mathrm{g})$, imipenem $(10 \mu \mathrm{g})$, meropenem $(10 \mu \mathrm{g})$, and colistin. For Gram-positive organisms, the antibiotics to be tested and reported were gentamicin $(10 \mu \mathrm{g})$, erythromycin $(15 \mu \mathrm{g})$, ciprofloxacin $(5 \mu \mathrm{g})$, oxacillin $(1 \mu \mathrm{g})$, ampicillin $(10 \mu \mathrm{g})$, clindamycin $(2 \mu \mathrm{g})$, vancomycin $(30 \mu \mathrm{g})$, co-trimoxazole $(1.25 / 23.75 \mathrm{Mg})$, doxycycline $(30 \mu \mathrm{g})$, teicoplanin $(30 \mu \mathrm{g})$, and linezolid $(30 \mu \mathrm{g})$.

The data regarding culture and sensitivity of the organisms isolated from different sources were retrieved from the electronic medical records.

\section{Statistical Analysis}

Categorical variables were described as frequency and percentage, and continuous variables as median and interquartile range. For continuous variables, mean values were compared using two sample t-tests for independent samples. Differences in proportions were compared using a Chi-square test or Fisher's exact test, as appropriate. $p$ value of $<0.05$ was considered statistically significant. The analyses were performed using SPSS software.

\section{Results}

All samples were collected as per standard institutional protocol ensuring complete asepsis during collection and handling.

The culture reports of 644 samples from medical oncology (425), surgical oncology (152) and radiation oncology (67) collected during the period from October 2016 to September 2017 were retrospectively analyzed.

Out of these 644 samples, 317 were blood samples, 148 were urine samples, 92 were tracheal samples, 44 were pus samples and 44 were other (sputum, stool, pleural, bronchoalveolar lavage) samples (Table 1).

A total of $31.33 \%(107 / 644)$ samples were positive for growth of organisms. In this positive culture samples, 76.63\% (82/107) were bacterial and $23.3 \%(25 / 107)$ were fungal. In the positive bacterial cultures, $84.14 \%$ (69/82) were Gram-negative and 15.86\% (13/82) were Gram-positive (Tables 2 and 3).

Among the Gram-negatives, the most prevalent organisms isolated were Klebsiella (24; 34.78\%) followed by Pseudomonas (15; 21.73\%), Acinetobacter spp. $(14 ; 20.28 \%)$ and Escherichia coli (13; 18.84\%). Among the Gram-positives, the most prevalent organisms isolated were Enterococcus spp. $(8 ; 61.53 \%)$ followed by Staphylococcus aureus (5; 38.46\%) (Tables 2 and 3 ).

Staphylococcus spp. were sensitive to vancomycin, amikacin and Linezolid and all strains were resistant to penicillins. All strains of Enterococcus spp. were sensitive to linezolid and resistant to penicillins and ciprofloxacin (Fig. 1).

Gram-negative organisms were all susceptible to colistin (100\%) and resistant in varying degrees to ciprofloxacin and ceftazidime. Susceptibility to levofloxacin was different for these organisms.

Table 1: Distribution of samples taken from patients treated at various oncology department

\begin{tabular}{|c|c|c|c|c|}
\hline Sample & Medical oncology & Radiation oncology & Surgical oncology & Total \\
\hline Blood & 226 & 37 & 54 & 317 \\
\hline Urine & 98 & 16 & 34 & 148 \\
\hline Tracheal & 57 & 11 & 24 & 92 \\
\hline Sputum & 13 & 2 & 2 & 17 \\
\hline Stool & 7 & & & 7 \\
\hline Pus/Wound & 9 & 1 & 34 & 44 \\
\hline Pleural & 8 & & 4 & 12 \\
\hline BAL & 7 & & & 7 \\
\hline Total & 425 & 67 & 152 & 644 \\
\hline
\end{tabular}

BAL: Bronchoalveolar lavage 
Table 2: Organism profiles in patients treated at various oncology department

\begin{tabular}{|c|c|c|c|c|}
\hline Organism & Medical oncology & Radiation oncology & Surgical oncology & Total \\
\hline \multicolumn{5}{|l|}{ Gram-positive } \\
\hline Staphylococcus aureus & & 1 & 4 & 5 \\
\hline Enterococcus & 4 & 1 & 3 & 8 \\
\hline \multicolumn{5}{|l|}{ Gram-negative } \\
\hline Escherichia coli & 8 & & 5 & 13 \\
\hline Klebsiella & 17 & 1 & 6 & 24 \\
\hline Pseudomonas & 11 & 1 & 3 & 15 \\
\hline Acinetobacter & 7 & 1 & 6 & 14 \\
\hline Citrobacter & & & 1 & 1 \\
\hline Burkholderia & 2 & & & 2 \\
\hline Total & 49 & 5 & 28 & 82 \\
\hline
\end{tabular}

Table 3: Organism profiles among various samples taken from patients

\begin{tabular}{|c|c|c|c|c|c|c|c|c|c|}
\hline Organism & Blood & Urine & Tracheal & Sputum & Stool & $\begin{array}{l}\text { Pus/ } \\
\text { wound }\end{array}$ & Pleural & $B A L$ & Total \\
\hline \multicolumn{10}{|l|}{ Gram-positive } \\
\hline Staphylococcus aureus & & & 1 & & & 4 & & & 5 \\
\hline Enterococcus & 6 & 1 & & & & 1 & & & 8 \\
\hline \multicolumn{10}{|l|}{ Gram-negative } \\
\hline Escherichia coli & 2 & 2 & & 2 & & 7 & & & 13 \\
\hline Klebsiella & 9 & 1 & 3 & 7 & & 3 & & 1 & 24 \\
\hline Pseudomonas & 4 & 1 & 4 & 3 & & 2 & & 1 & 15 \\
\hline Acinetobacter & 1 & & 9 & 2 & & 1 & & 1 & 14 \\
\hline Proteus & & & & & & & & & 0 \\
\hline Citrobacter & & & & & & 1 & & & 1 \\
\hline Burkholderia & 1 & & & & & & & 1 & 2 \\
\hline Fungal & & 25 & & & & & & & 25 \\
\hline
\end{tabular}

BAL: Bronchoalveolar lavage

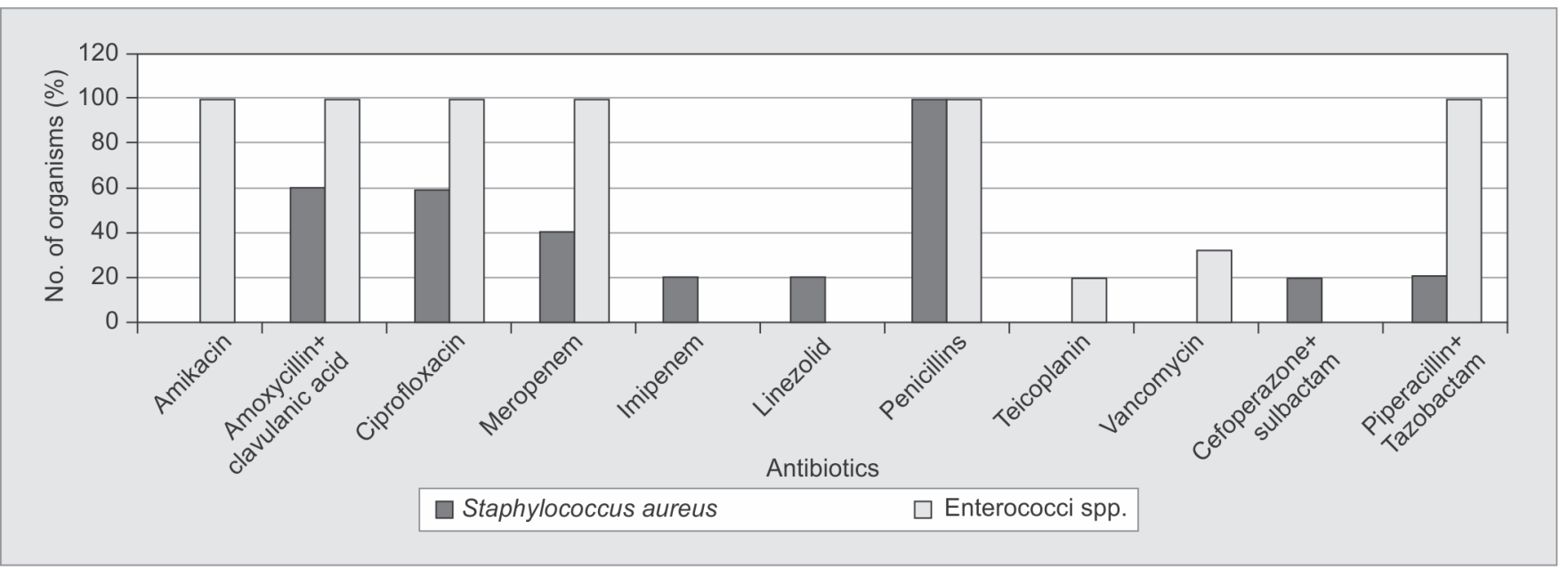

Fig. 1: Resistance patterns of Gram-positive organisms

Klebsiella and Pseudomonas spp. were resistant whereas E. coli and Acinetobacter spp were sensitive to levofloxacin (Fig. 2).

The antimicrobial sensitivity of organisms isolated from blood is shown in Figures $3 \mathrm{~A}$ and B. Major Gram-positive organisms were sensitive to antibiotics, such as vancomycin, teicoplanin and linezolid. Gram-negative isolates in the blood had about
$40-55 \%$ sensitivity to third generation cephalosporins. Among the $\beta$-lactam- $\beta$-lactamase inhibitor combination that is piperacillin and tazobactam [Pip-Taz], $67 \%$ of the $E$. coli isolates were sensitive as compared to only $15 \%$ and $40 \%$ of Klebsiella and Pseudomonas, respectively. All Gram-negative isolates were $100 \%$ sensitive to colistin. 


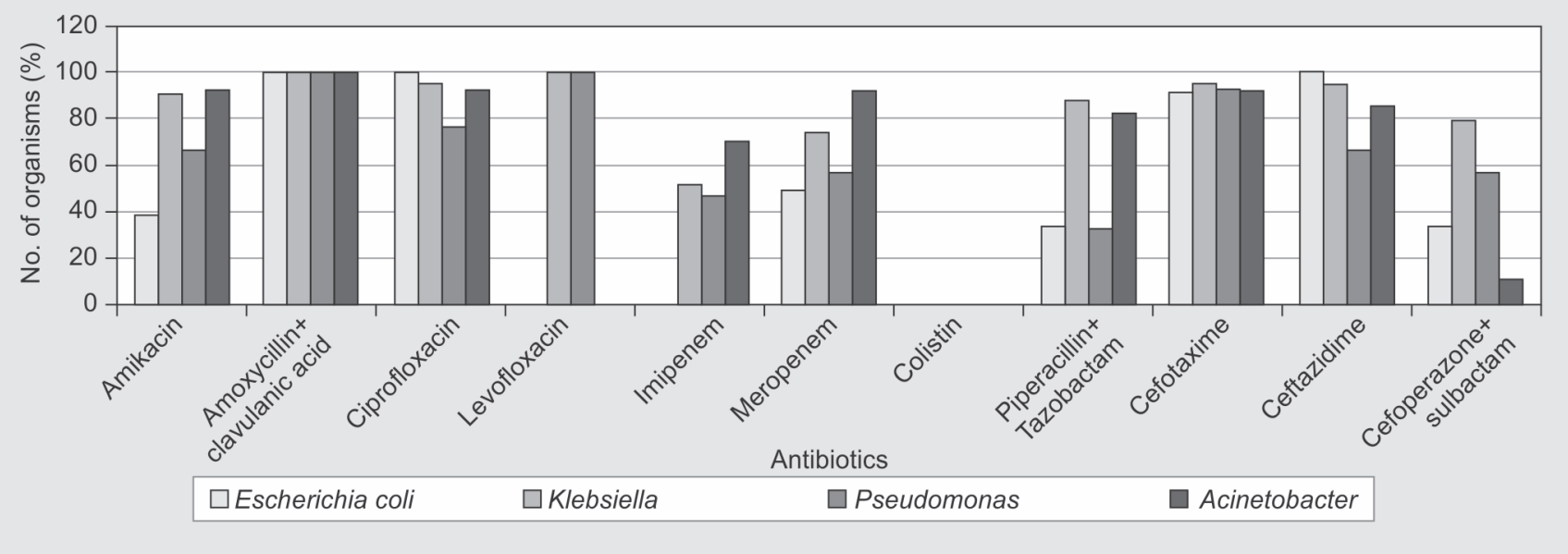

Fig. 2: Resistance patterns of Gram-negative organisms

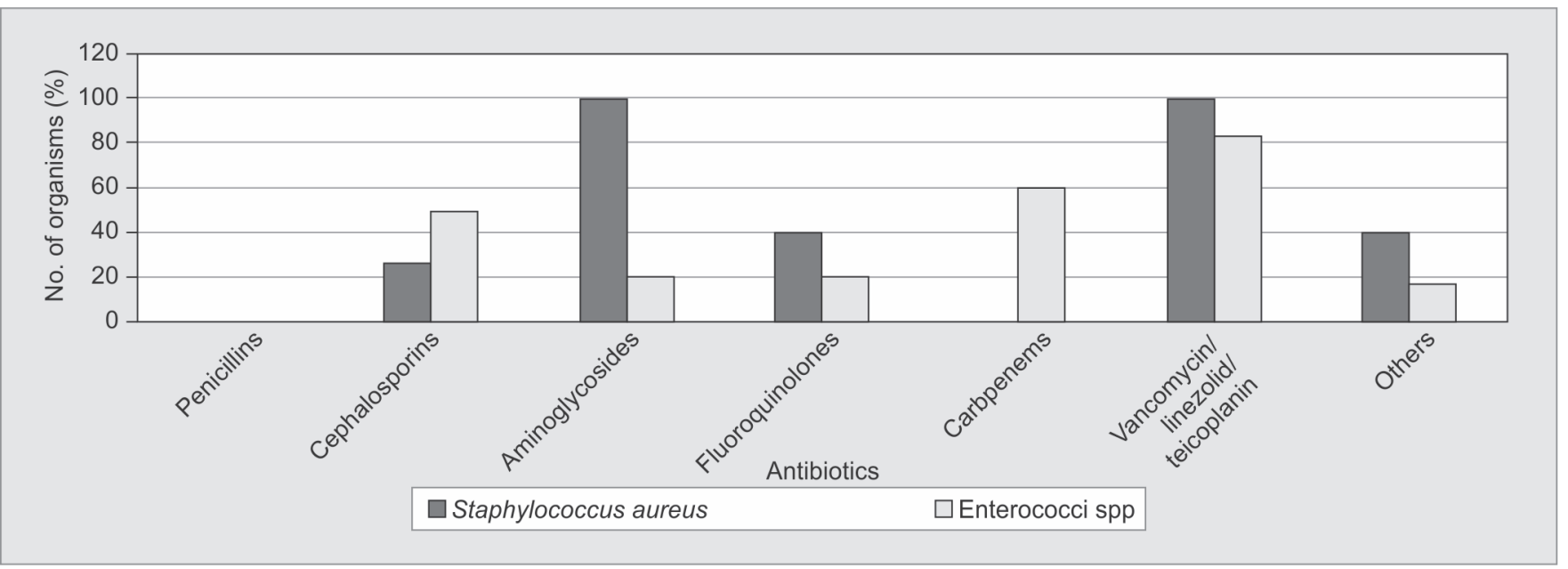

Fig. 3A: Common Gram-positive isolates sensitivity in blood

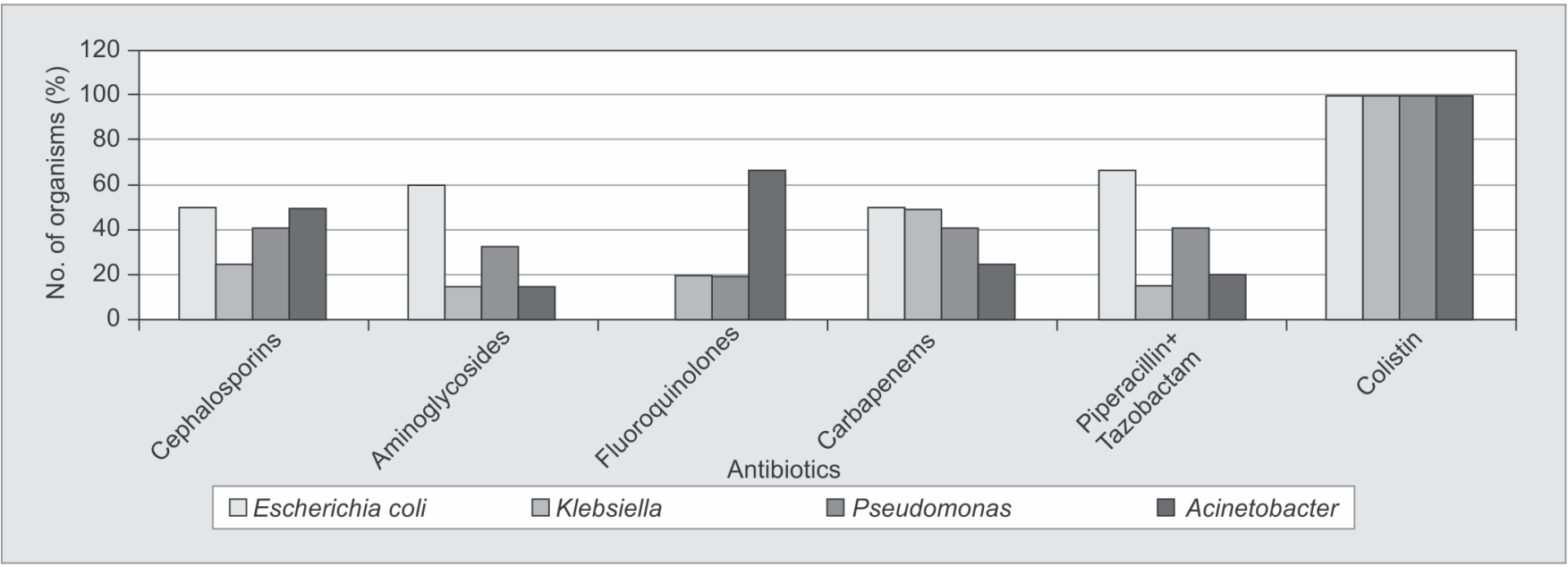

Fig. 3B: Common Gram-negative isolates sensitivity in blood 


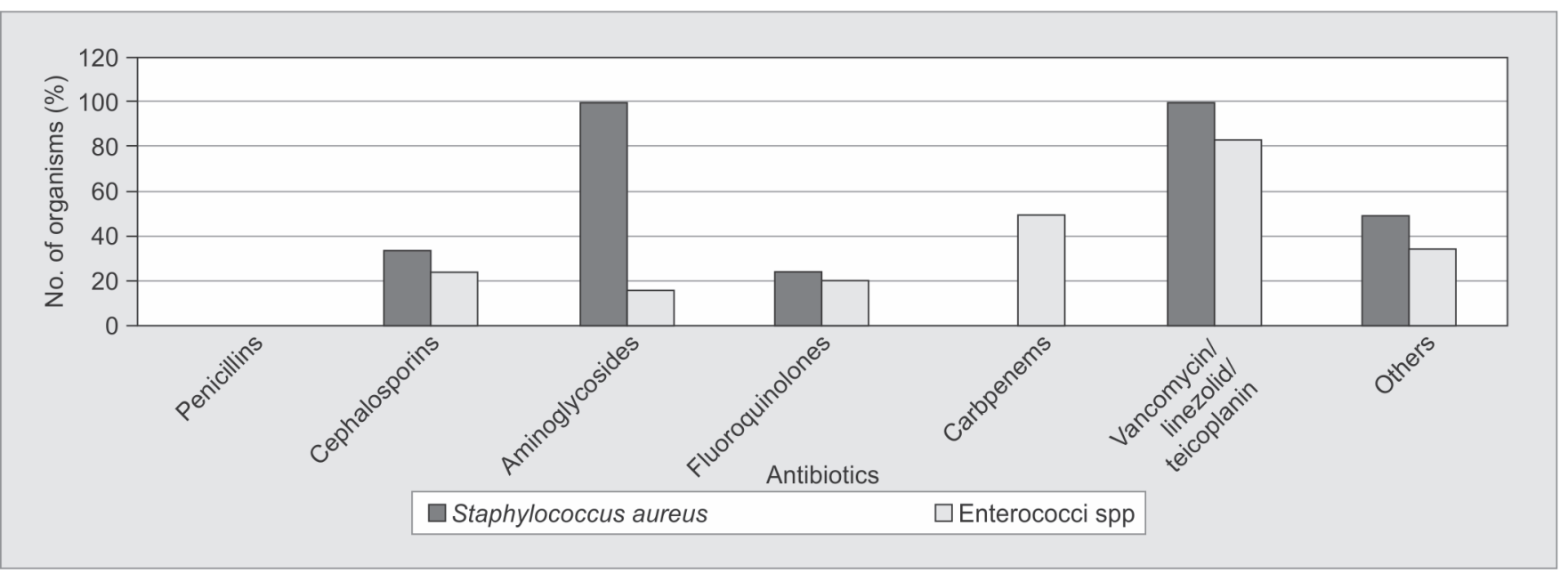

Fig. 4A: Common Gram-positive isolates sensitivity in respiratory isolates

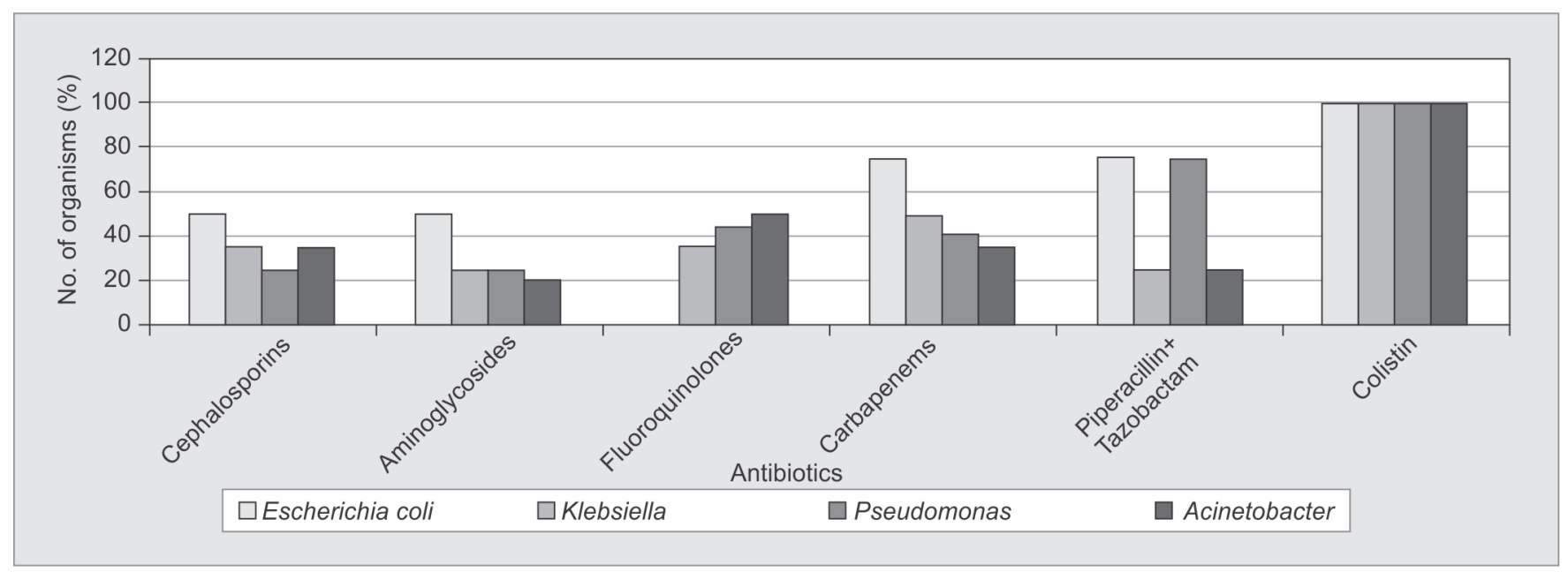

Figure 4B: Common Gram-negative isolates sensitivity in respiratory isolates

The antimicrobial sensitivity of organisms isolated from respiratory secretions is shown in Figures 4 A and B. S. aureus showed no sensitivity to penicillins but $100 \%$ sensitive to vancomycin, teicoplanin and linezolid. Almost $100 \%$ of various Gram-negative isolates were sensitive to colistin. All the Gram-negative isolates had sensitivity to third generation cephalosporins ranging from 25 to $50 \%$.

\section{Discussion}

Infections are common cause of morbidity and mortality in cancer patients. Various guidelines have recommended early antibiotic therapy on basis of local culture and sensitivity patterns. This prevents unnecessary antibiotics usage and emergence of drugresistant strains.

In our retrospective study, we have analyzed various cultures sent from patients admitted in our ICU at a tertiary care cancer center. We found that Gram-negative isolates were most common in our setup. This high prevalence of Gram-negative isolates has also been reported by various earlier studies in India in oncology centres ${ }^{10-13}$. Our study findings were also consistent with worldwide results where the predominance of Gram-negative isolates is common as reported in our study. In patients with cancer, the pattern of infections has shifted towards Gram-negative organisms from Gram-positive organisms in the recent years ${ }^{14-16}$. This may be attributed to infrequent use of indwelling catheters, less cytotoxic agents for chemotherapy and decreased use of antibiotic prophylaxis.

In our study, the prevalence of various organisms was as follows: Klebsiella (24/82, 29.26\%), Pseudomonas (15/82, 18.29\%), Acinetobacter spp. $(14 / 82,17.07 \%)$ and Enterococcus spp. $(8 / 82$, 9.75\%). Similar findings were also observed by Nazneen et al., a study conducted at cancer centre at Aurangabad, Marathwada ${ }^{12}$. Among Gram-negative isolates, Klebsiella (34.78\%) was the predominant organism followed by Pseudomonas (21.73\%) in our study. This was in contrast to Singh et al. study conducted at cancer center at Delhi where predominant organism was E. coli $(23.5 \%)$ with very low incidence of Pseudomonas (6.7\%). ${ }^{10}$ This may be due to different antibiotic prescription policies.

In this study, among Gram-positive isolates, Enterococcus spp. (61.53\%) was most commonly isolated followed by S. aureus (38.46 $\%)$. This was rather different from prevalence rates in most other studies done in cancer population where predominant Grampositive organism was $S$. aureus ${ }^{10,12,17}$. 
Most of the $E$. coli and $K$. pneumoniae isolates were resistant to the third generation Cephalosporins (cefotaxime/ceftazidime) and $\beta$-lactam/ $\beta$-lactamase inhibitor combinations, such as cefoperazone-sulbactam and Pip-Taz. Similar high rates of resistance of these organisms to the third generation cephalosporins have been noted in a study from Mumbai by Bhat et $a l^{11}$. and another study from Aurangabad by Nazneen et al ${ }^{12}$. However more than $50 \%$ of K. pneumoniae and Acinetobacter isolates were resistant to amikacin, ciprofloxacin.

In our study, the resistance of Acinetobacter spp. to aminoglycosides and carbapenems was quite significant. Very high rates of resistance of acinetobacter to ceftazidime (84\%), ciprofloxacin (60\%) and imipenem (35\%) was showed in one study from Delhi, India conducted by Ghosh et al. ${ }^{18}$ at a tertiary care cancer center.

The magnitude of antibiotic resistance is fortunately not as high among the Gram-positive compared to Gram-negative organisms. High rates of methicillin resistance [Delhi (35\%), Mumbai (42\%)] 9,10 and the emergence of vancomycin intermediate strains of $S$. aureus have also been reported from India. However we did not encounter any resistance to vancomycin among Staphylococcus.

This high prevalence of resistant organisms highlights the importance of formulating antibiotic policies based on local antibiotic susceptibility patterns so that arbitrary use of antibiotics is avoided and resistance is kept to a minimum.

\section{Limitations}

Our study has certain limitations. First, it was conducted in a single institution, which may not demonstrate the epidemiology of different centres or different geographical areas. Second, this study provides one-time information about the antibiotic sensitivity which is not sufficient, as the periodic revision of the sensitivity pattern is very essential. Third, being a retrospective study, we did not have the details of the timing of samples sent whether before or on antibiotics, underlying disease and previous antibiotic exposure as these parameters may have different epidemiologic, clinical characteristics, and microbial pattern.

\section{Conclusion}

Our study highlights that an antibiogram for ICU patients may help the clinician to understand local susceptibility patterns and help them to make an informed decision about the initial empirical antibiotic.

Regular monitoring of the pattern of resistance of bacteriological isolates in cancer patients is critical to develop much needed antibiotic policy to combat these infections early. Continuous antibiotic stewardship is required and should be monitored on regular basis to improve outcomes.

\section{References}

1. Kamboj M, Sepkowitz KA. Nosocomial infections in patients with cancer. Lancet Oncol 2009;10:589-597.

2. Cornejo-Juárez P, Vilar-Compte $D$, et al. The impact of hospitalacquired infections with multidrug-resistant bacteria in an oncology intensive care unit. Int J Infect Dis 2015;31:31-34.

3. Barai L, Fatema K, et al. Bacterial profile and their antimicrobial resistance pattern in an intensive care unit of a tertiary care hospital in Dhaka. Ibrahim Med Coll J 2010;4:66-69.

4. Günseren F, Mamikoğlu L, et al. A surveillance study of antimicrobial resistance of gram-negative bacteria isolated from intensive care units in eight hospitals in Turkey. J Antimicrob Chemother 1999;43:373-378.

5. Gudiol C, Carratalà J. Antibiotic resistance in cancer patients. Expert Rev Anti Infect Ther 2014;12:1003-1016.

6. Basak S, Singh $P$, et al. Multidrug Resistant and Extensively Drug Resistant Bacteria: A Study. Journal of Pathogens. 2016; 2016:4065603.

7. Clinical and Laboratory Standards Institute. Performance Standards for Antimicrobial Susceptibility Testing. Twentysecond Informational Supplement (M100-S22). Wayne, PA, USA: CLSI; 2016.

8. Bauer AW, Kirby WM, et al. Antibiotic susceptibility testing by a standardized single disk method. Am J Clin Pathol 1966;45:4936.

9. European Committee on Antimicrobial Susceptibility Testing (EUCAST). Breakpoint tables for interpretation of MICs and zone diameters. Ver. 6.0, 2016. EUCAST; 2016.

10. Singh R, Jain $\mathrm{S}$, et al. Characterization and antimicrobial susceptibility of bacterial isolates: Experience from a tertiary care cancer centre in Delhi. Indian J Cancer 2014;51:47780.

11. Bhat V, Gupta S, et al. Bacteriological profile and antibiotic susceptibility patterns of clinical isolates in a tertiary care cancer centre. Indian J Med Paediatr Oncol 2016;37:204.

12. Nazneen S, Mukta K, et al. Bacteriological trends and antibiotic susceptibility patterns of clinical isolates at Government Cancer Hospital, Marathwada. Indian J Cancer 2016;53:583-586.

13. Karanwal AB, Parikh BJ, et al. Review of clinical profile and bacterial spectrum and sensitivity patterns of pathogens in febrile neutropenic patients in hematological malignancies: A retrospective analysis from a single center. Indian J Med Paediatr Oncol 2013;34:858.

14. Montassier $E$, Batard $E$, et al., de La Cochetière MF. Recent changes in bacteremia in patients with cancer: a systematic review of epidemiology and antibiotic resistance. Eur J Clin Microbiol Infect Dis 2013;32:841-850.

15. Gudiol C, Aguado JM, et al. Bloodstream infections in patients with solid tumors. Virulence 2016;7:298-308.

16. Royo-Cebrecos C, Gudiol C, et al. A fresh look at polymicrobial bloodstream infection in cancer patients. PLoS ONE 2017;12(10):e0185768.

17. Prabhash $\mathrm{K}$, Medhekar A, et al. Blood stream infections in cancer patients: a single center experience of isolates and sensitivity pattern. Indian J Cancer 2010;47:1848.

18. Ghosh I, Raina V, et al. Profile of infections and outcome in high-risk febrile neutropenia: experience from a tertiary care cancer center in India. Med Oncol Northwood Lond Engl 2012;29:1354-1360. 